ARTICLE

https://doi.org/10.1038/s41467-021-25940-6

\title{
Brain adiponectin signaling controls peripheral insulin response in Drosophila
}

Nathalie Arquier (10 ${ }^{1 凶}$, Marianne Bjordal ${ }^{2}$, Philippe Hammann (10 ${ }^{3}$, Lauriane Kuhn (10 ${ }^{3}$ \& Pierre Léopold ${ }^{4}$

The brain plays a key role in energy homeostasis, detecting nutrients, metabolites and circulating hormones from peripheral organs and integrating this information to control food intake and energy expenditure. Here, we show that a group of neurons in the Drosophila larval brain expresses the adiponectin receptor (AdipoR) and controls systemic growth and metabolism through insulin signaling. We identify glucose-regulated protein 78 (Grp78) as a circulating antagonist of AdipoR function produced by fat cells in response to dietary sugar. We further show that central AdipoR signaling inhibits peripheral Juvenile Hormone $(\mathrm{JH})$ response, promoting insulin signaling. In conclusion, we identify a neuroendocrine axis whereby AdipoR-positive neurons control systemic insulin response.

\footnotetext{
${ }^{1}$ Aix-Marseille University, INSERM, TAGC, UMR_S1090, Marseille, France. ${ }^{2}$ Université Côte d'Azur, CNRS UMR7277, Inserm U1091, iBV, Parc Valrose, 06108 Nice, France. ${ }^{3}$ Plateforme Protéomique Strasbourg-Esplanade FRC 1589, University of Strasbourg, CNRS, 15 rue René Descartes, 67084 Strasbourg cedex, France. ${ }^{4}$ Institut Curie, PSL Research University, CNRS UMR3215, INSERM U934, 26 Rue d'Ulm, 75005 Paris, France. ${ }^{凶}$ email: nathalie.arquier@univamu.fr
} 
E nergy homeostasis is controlled by a complex interplay between peripheral organs and the brain. In the vertebrate brain, nuclei within the hypothalamus crosstalk and integrate peripheral signals such as the level of adiposity and caloric intake to regulate food intake and energy expenditure ${ }^{1}$. Glucose being the primary source of energy, its availability is constantly monitored through sensor and effector mechanisms ${ }^{2}$. Adiponectin is an abundant adipokine present in the bloodstream of mice and humans, whose levels inversely correlate with circulating glucose ${ }^{3}$. Adiponectin receptor expression also inversely correlates with insulinemic and glycemic states in mice, suggesting that adiponectin signaling could serve as a marker for insulin sensitivity. In line with this, Adiponectin knockout mice fed a high-fat/high-sucrose diet develop severe insulin resistance ${ }^{5}$. Adiponectin can cross the blood-brain barrier ${ }^{6,7}$, and one isoform of its receptor, AdipoR1, is expressed in the hypothalamus in humans ${ }^{8}$. However, conflicting reports have confounded the evaluation of the role of brain adiponectin signaling in energy homeostasis. Our current study using Drosophila identifies crosstalk between the brain and peripheral organs whereby adiponectin receptor-positive neurons receive nutritional inputs and control general insulin signaling by modulating the levels of peripheral juvenile hormone $(\mathrm{JH})$ response.

\section{Results and discussion}

Brain adiponectin receptor controls animal growth. Drosophila has a single adiponectin receptor, AdipoR, encoded by gene CG5315 (referred to as AdipoR). Independent batches of AdipoR antibodies labeled the same symmetrical cluster of neurons in the larval brain lobes only (Fig. 1a and Supplementary Fig. 1a-e), referred to as adiponectin receptor-positive neurons (APNs). Unlike a previous report ${ }^{9}$, our anti-AdipoR antibodies did not label the brain insulin-producing cells (IPCs) (Supplementary Fig. 1b). The pattern of Gal4 expression of Janelia GAL4 line \#48522 (see Methods), was found to overlap to a large extent with the APNs (Supplementary Fig. 1a) and was further referred to as Apn-GAL4 (Apn >). RNAi-mediated silencing of AdipoR in APNs using two independent RNAi lines $(A p n>A d i p o R-R i$ and Apn $>$ AdipoR-TRIP) induced partial larval lethality with a reduced imaginal disc ( $-48 \%$, Fig. 1b) and larval body growth (Supplementary Fig. 1f). Emerging adults had smaller wings $(-10 \%$, Fig. 1c and Supplementary Fig. 1g) and reduced weight $(-20 \%$, Fig. 1d and Supplementary Fig. 1h), accompanied with a $63 \%$ reduction of anti-AdipoR staining in the APNs (Supplementary Fig. 1c- $\left.c^{\prime}\right)$. Reducing AdipoR in the IPCs had no effect on growth (dilp2 $>$ AdipoR-Ri; Supplementary Fig. 1i) suggesting an effect on growth control independent of IPC function. Activating AdipoR in the APNs induced growth increase $(+12 \%$, Fig. 1d, Apn $>$ AdipoR-act, see Supplementary materials for details). Similarly, expression of human adiponectin (hAdipoQ), the vertebrate AdipoR agonist, from larval fat cells or APNs, induced systemic growth with adults heavier than controls (lpp $>h$ AdipoQ, $+7 \%$; Fig. 1d; Apn $>h A d i p o Q,+14 \%$, Supplementary Fig. 1k). Moreover, a strong hypomorphic mutant for AdipoR (AdipoR ${ }^{M}$, Supplementary Fig. 1n) exhibited reduced larval growth (Supplementary Fig. $1 \mathrm{~m}$ ) and developmental lethality in accordance with the Apn $>$ AdipoR-Ri phenotypes. Reducing AdipoR function in the larval fat body or in the muscles, two organs with adiponectin signaling function in vertebrates ${ }^{10}$, induced a moderate increase in adult size, which did not account for the AdipoR ${ }^{M}$ phenotype (Supplementary Fig. 11). Collectively, these results indicate that Drosophila AdipoR acts in the brain to control systemic growth. a

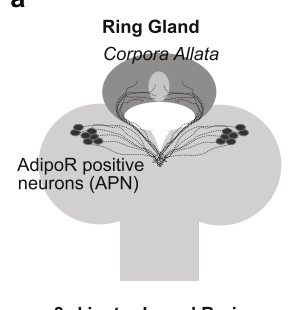

3rd instar Larval Brain
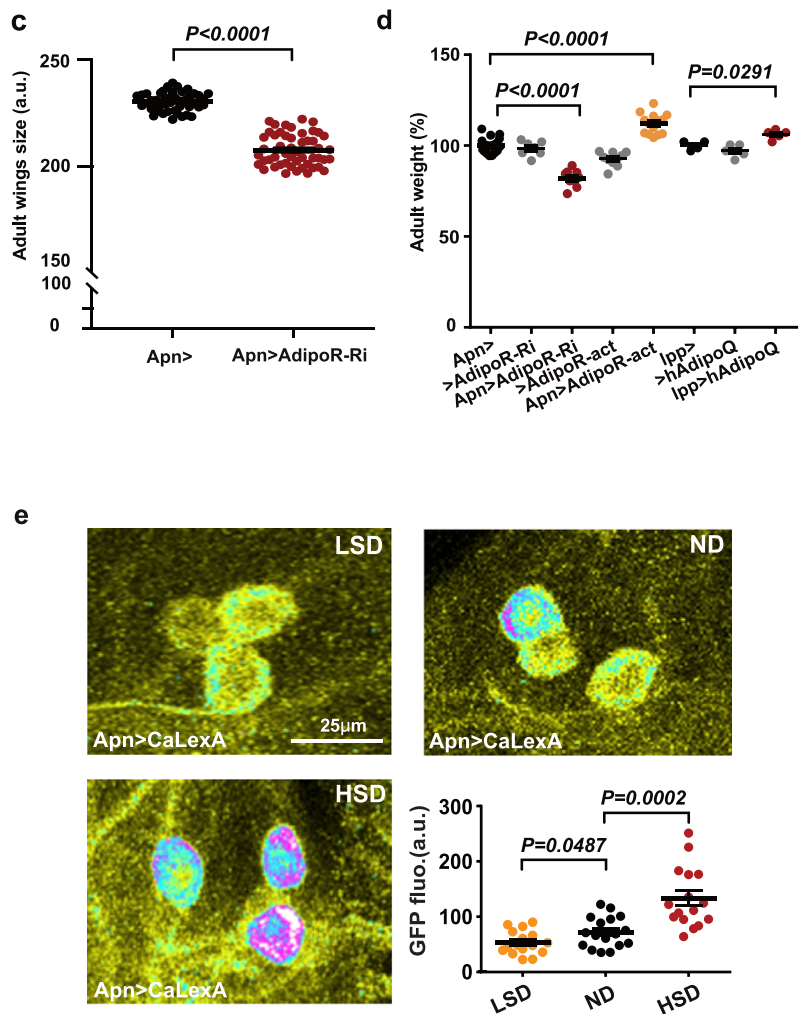

f
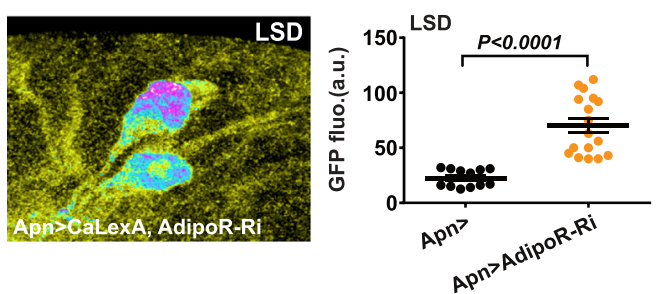

g
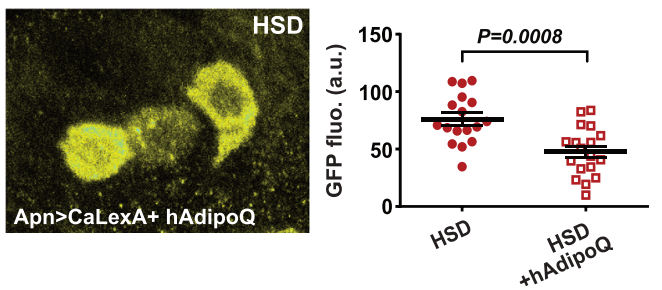

AdipoR controls APN activity according to dietary sugars. The role of vertebrate AdipoR signaling in energy homeostasis suggested that the fly APNs could function in sensing food caloric content. Using the calcium reporter UAS-CaLexA as a marker for synaptic activity ${ }^{11}$, we observed that activation of APNs gradually increased with dietary sugar content (Fig. 1e). In addition, silencing AdipoR in APNs increased neuronal activity in larvae 
Fig. 1 Central AdipoR function controls animal growth. a Scheme of AdipoR-positive neurons (APNs) and their projections on the corpora allata of the ring gland. b Measure of larval wing disc size $96 \mathrm{~h}$ after egg laying (AEL) from Apn > AdipoR-Ri ( $n=45$, red) and $A p n>(n=52$, black $)$. c Measure of adult wing size of $A p n>\operatorname{AdipoR-Ri}(n=51$, red) and Apn $>(n=47$, black). $n=$ biologically independent samples from five (b) and three (c) independent experiments. Statistical significance was tested using ordinary two-way ANOVA with Sidak's multiple comparisons test. d Measure of adult weight of $A p n>\operatorname{AdipoR-Ri}(n=9$, red), $A p n>(n=20$, black), $>$ AdipoR-Ri $(n=9$, gray), $>$ AdipoR-act $(n=10$, gray), Apn $>$ AdipoRact $(n=14$, orange), Ipp $>(n=4$, black), $>h A d i p o Q ~(n=5$, gray) and lpp $>$ hAdipoQ $(n=5$, red). $n=$ groups of 5-10 males. Statistical significance was tested ordinary one-way ANOVA with Tukey's multiple comparisons test. e APN activity levels at $96 \mathrm{~h}$ AEL revealed by anti-GFP immunostaining and quantified by GFP fluorescence intensity using a UAS-CaLexA probe (see Methods) in different sugar diets (LSD, ND, HSD), upon modulation of AdipoR expression in LSD (in $\mathbf{f}$ ) or upon human AdipoQ feeding (in $\mathbf{g}$ ). Quantifications are shown in accompanying panels (e, LSD $n=16$, orange; ND $n=17$, black; HSD $n=16$, red), (f, Apn > AdipoR-Ri $n=17$, orange; Apn $>n=12$, black), $(\mathbf{g}$; hAdipoQ $n=20$, red squares; control $n=18$, red points). $n=$ number of independent GFP measurements. Statistical significance was tested using a two-sided Mann-Whitney test. Data were presented as mean values \pm SEM. $P$ values are indicated in all panels. a.u. arbitrary units. Source data are provided as a Source Data file.

fed a low-sugar diet (LSD) (Fig. 1f), whereas feeding larvae on a high-sugar diet (HSD) supplemented with human adiponectin (+hAdipoQ) prevented APNs activation (Fig. 1g). We concluded that AdipoR function blocks APNs activity and that human adiponectin acts as an agonist of the fly AdipoR (Fig. 1d and Supplementary Fig. 1k) to prevent APNs activation by dietary sugar. Therefore, an endogenous agonist of AdipoR could be produced in LSD, maintaining APNs silent, or AdipoR could be repressed by an antagonist partner in response to HSD leading to APN activation, or both regulations could take place at various dietary sugar levels.

Grp78 is an antagonist partner of AdipoR. The fly genome does not encode a peptide with adiponectin-related sequences. Therefore, we conducted IP-mass spectrometry (IP-MS) on larvae ubiquitously expressing Myc-tagged AdipoR from a tubulin promoter $(t u b>$ AdipoR-Myc) to identify endogenous interactors of AdipoR. Following anti-Myc immunoprecipitation, larval lysate was subjected to MS analysis and AdipoR-associated proteins were identified (Supplementary Fig. 2a, b and Supplementary Materials). IP-MS specificity was tested by mock IP with larval extracts expressing an unrelated seven-pass membraneassociated protein (Smo-myc; see Methods). Genes encoding secreted or putative secreted products were validated by knockdown in fat cells, and pupal lethality was scored (Supplementary Fig. 2c and Supplementary Materials). Among the putative candidates, glucose-regulated protein 78 (Grp78, also named BIP or HSC70-3), is regulated by sugar both in mammals and flies ${ }^{12,13}$. Antibodies raised against the mammalian homolog ${ }^{12}$ detected an increase of Grp78 amount in the hemolymph of larvae raised on HSD compared to LSD (Fig. 2a and Supplementary Fig. 2d, e). Grp78 is a component of the unfolded protein response (UPR) ${ }^{14}$. However, examining $X b p 1$ splicing and its nuclear localization in fat body cells as markers of UPR, we found that UPR is not activated in our HSD conditions (Supplementary Fig. 2f-h), suggesting that Grp78 acts on adiponectin signaling independently of UPR. grp78 mRNA levels were not modified by our dietary sugar conditions (Fig. 2b), in line with a regulation of Grp78 function independent of UPR and ATF6 transcriptional activation $^{15}$. Reciprocal co-immunoprecipitation experiments in S2 cells also suggested specific interactions between AdipoR, hAdipoQ, and Grp78 (Supplementary Fig. 1j, Fig. 2c). Finally, when dissected brains from larvae fed on LSD were cultured in the presence of hemolymph from larvae fed on HSD, increased APNs activity was observed (Fig. $2 \mathrm{~d}-\mathrm{d}^{\prime}$ ), providing an ex-vivo confirmation of in vivo results (Fig. 1e). However, increased APNs activity was not observed when hemolymph was collected from larvae fed on HSD with fat body-specific grp78 silencing (lpp $>$ grp78-Ri, Fig. $\left.2 \mathrm{~d}-\mathrm{d}^{\prime}\right)$. This indicates that Grp78 is an antagonistic partner of AdipoR produced by the adipose tissue and required for APNs activation in high sugar conditions.

Antagonistic interaction between AdipoR and JH signaling. APNs send numerous projections towards a key endocrine organ called the ring gland (RG) (Fig. 3a), which produces three metabolic hormones, adipokinetic hormone $(\mathrm{AKH}), \mathrm{JH}$, and ecdysone. We then examined the role of these hormones as a potential peripheral effector for the central function of AdipoR. Silencing AdipoR in APNs $(A p n>A d i p o R-R i)$ resulted in increased expression of $\mathrm{JH}$-inducible protein 26 (jhi-26), a target of $\mathrm{JH}_{\text {signaling }}{ }^{16}$ (Fig. 3b). A similar result was observed for $\mathrm{Kr}$ $\mathrm{h} 1$, the transcriptional effector for $\mathrm{JH}$ signaling (Fig. 3c). This suggested that inhibiting AdipoR in the APNs leads to elevated $\mathrm{JH}$ signaling. In this line, as observed for $A p n>A d i p o R-R i$ animals, $w t$ larvae fed with pyriproxyfen, a potent $\mathrm{JH}$ analog $(\mathrm{JHa})^{17}$, grew slowly ( $-69 \%$ in imaginal wing disc size, Fig. $3 \mathrm{~d})$ and adult escapers exhibited weight reduction ( $-6 \%$, Fig. 3e). Hemizygous females mutant for both met and gce (encoding the two $\mathrm{JH}$ receptors in Drosophila ${ }^{18}$ ), showed survival rates similar to controls (+/FM7; Apn > compared to met $^{27}, g c e^{25 k} / F M 7 ; ; A p n>$; see Fig. 3f). However, introducing hemyzygous $\mathrm{met}^{27}, \mathrm{gce}^{25 k}$ mutations ( $m e t^{27}, g c e^{25 k} / \mathrm{FM} 7$ females) efficiently rescued the lethality and the size of $A p n>A d i p o R-R i$ animals (Fig. 3f, g), indicative of a genetic interaction between Adipo $\mathrm{R}$ and $\mathrm{JH}$ signaling. Although a direct effect of brain AdipoR on peripheral $\mathrm{JH}$ targets is possible, our data suggest an antagonistic interaction between brain AdipoR signaling and $\mathrm{JH}$ production and signaling.

No difference was detected in $A k h$ mRNA level or protein level in the RG of $A p n>A d i p o R-R i$ larvae (Supplementary Fig. 3a, c), suggesting that $\mathrm{AKH}$ signaling is not majorly affected by AdipoR signaling. Moreover, APNs neuronal projections are distinct from those of AKH-producing cells (Supplementary Fig. 3b). Analysing ecdysone signaling, we found that expression of E78, an ecdysone receptor target, is delayed in $A p n>A d i p o R-R i$ larvae (Supplementary Fig. 3d). This correlated with delayed pupariation $\left(+12 \mathrm{~h}\right.$ at $29^{\circ} \mathrm{C}$; Supplementary Fig. $\left.3 \mathrm{e}\right)$ and the delayed accumulation of circulating and total levels of $20 \mathrm{E}$ (the active form of ecdysone). The same delays were observed in JHa-fed animals (Supplementary Fig. 3f-h). However, AdipoR is not present in PTTH-producing neurons (Supplementary Fig. 3i) and the mRNA levels of $p t t h$ are not altered in Apn $>A d i p o R-R i$ larvae (Supplementary Fig. 3j). In addition, $A p n>A d i p o R-R i$ larvae are significantly smaller than control animals, in contrast to ptth mutant larvae, which grow to larger size ${ }^{19,20}$. Finally, feeding Apn $>$ AdipoR-Ri animals with $20 \mathrm{E}$ did not rescue their lethality (Supplementary Fig. 3k). These results indicate that while ecdysone production is defective in $A p n>A d i p o R-R i$ larvae, this appears to be a consequence of increased $\mathrm{JH}$ production.

Brain AdipoR regulates peripheral insulin sensitivity. In light of the growth phenotypes, we examined the role of brain AdipoR in modulating peripheral insulin response. Using a tubulin::GFP$P H(t G P H)$ construct as an indicator of PI3-kinase activity ${ }^{21}$, we observed a reduction of basal insulin signaling in the fat bodies of 
a

hemolymph

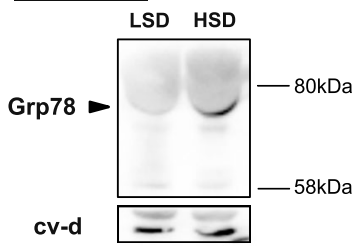

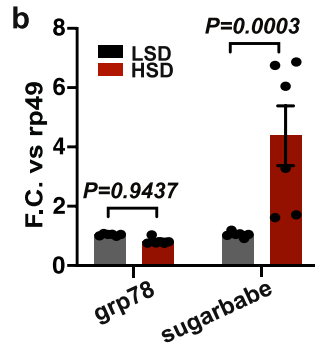
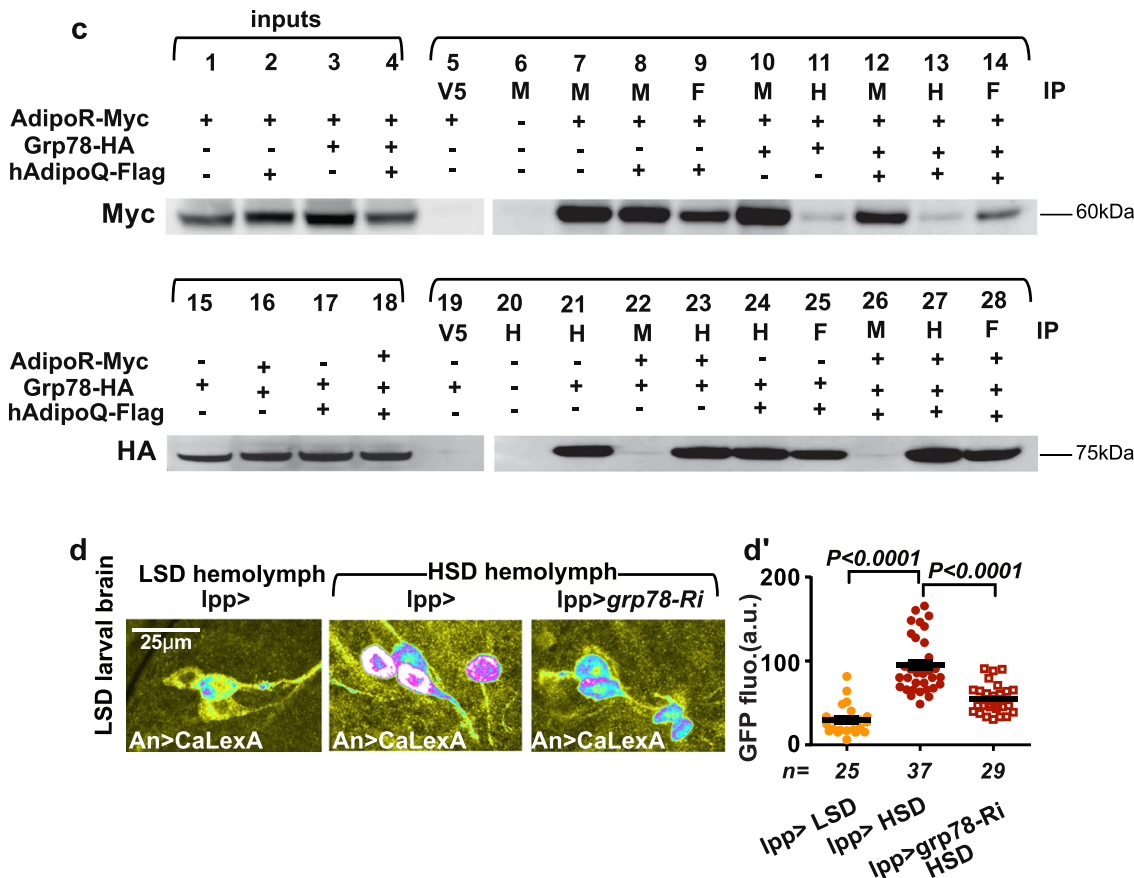

Fig. 2 Grp78 is a sugar-induced inhibitory partner for AdipoR. a Grp78 immunodetection in the hemolymph of low (LSD) and high (HSD) sugar diet-fed larvae. CV-d is a control for hemolymph proteins. (the experiment was repeated three times independently with similar results). b grp78 RNA levels in the whole larva in LSD (grey) versus HSD (red). QRT-PCR profiles, fold changes (FC) normalized to rp49. sugarbabe serves as a control for sugar induction. Statistical significance was tested using ordinary two-way ANOVA with Sidak's multiple comparisons test. $n=3$ biologically independent replicates per experiment (two independent experiments). c Co-immunoprecipitations (co-IP) of AdipoR-Myc, Grp78-HA, and hAdipoQ-Flag expressed in S2 cells. Inputs (lanes 1-4; 15-18). Myc (M), HA (H), and Flag (F) IPs are used to visualize AdipoR-Myc (5-14) or Grp78-HA (19-28). (the experiment was repeated three times independently with similar results). $\mathbf{d}$ Brain from Apn > CaLexA larvae fed in LSD and incubated with the hemolymph from Ipp $>$ control larvae (LSD- $n=25$, orange or HSD-fed $n=37$, red points) or Ipp > grp78-Ri larvae ( $-40 \%$; HSD-fed $n=29$, red squares). d' Quantification of GFP fluorescence in APN. $n=$ independent averaged GFP intensity measurements in APN. Statistical significance was tested using a two-sided Mann-Whitney test. Data were presented as mean values \pm SEM. $P$ values are indicated in all panels. a.u. arbitrary units. Source data are provided as a Source Data file.

Apn $>$ AdipoR-Ri larvae (Supplementary Fig. 4b). Then, we tested the ability of larval fat body explants to respond to human insulin in different genetic conditions. Compared to control explants, Apn $>$ AdipoR-Ri fat bodies showed limited ability to activate PI3K after exposure to insulin (Fig. $\left.4 a, a^{\prime}\right)^{22,23}$. In addition, insulinemia, measured as circulating levels of Drosophila insulinlike peptide 2 (Dilp2) in hemolymph, was found increased in Apn $>$ AdipoR-Ri and Apn > AdipoR-TRIP larvae (+55\% and 19\% respectively, Fig. $4 \mathrm{c}$ and Supplementary Fig. $4 \mathrm{f})$. Conversely, the expression of an activated form of AdipoR (AdipoR-act) leads to a substantial decrease in circulating Dilp2 (Fig. 4c). Production of human AdipoQ in the larval fat body ( $l p p>h A d i p o Q)$ lead to a similar decrease in insulinemia (Fig. 4d). This occurred without a change in dilp2 transcription (Supplementary Fig. 4d). Notably, silencing AdipoR in the IPCs had no incidence on circulating Dilp2 levels (Supplementary Fig. 4e). When challenged for glucose tolerance (ref. ${ }^{24}$ and Supplementary Materials for details), Apn $>$ AdipoR-RNAi larvae did not clear glucose from hemolymph as efficiently as controls (Fig. 4f), confirming a state of insulin resistance. In line with this, mRNA levels for the
Drosophila insulin receptor gene (Inr) were found reduced in Apn $>$ AdipoR-Ri larvae (Fig. 4g), as previously described in mouse models of insulin resistance ${ }^{25}$.

Insulin sensitivity is affected by $\mathrm{JH}$ signaling and Grp78. Our finding that central AdipoR signaling controls peripheral $\mathrm{JH}$ targets suggested that circulating $\mathrm{JH}$ modulates insulin-response in peripheral tissues. Indeed, feeding larvae with $\mathrm{JHa}$ increased circulating Dilp2 levels compared to control-fed animals (Fig. 4e). In addition, when pretreated with $\mathrm{JHa}$, fat body explants showed a reduced ability to respond to insulin stimulation (Fig. $4 \mathrm{~b}$ and Supplementary Fig. 4c). In line with this, Inr transcription was reduced in fat body cells treated with $\mathrm{JHa}$ and did not vary significantly after insulin stimulation (Supplementary Fig. 4a). Therefore, JH signaling in peripheral tissues directly inhibits local insulin signaling. These observations are in line with the recent finding that $\mathrm{Kr}-\mathrm{h} 1$, the transcriptional effector of $\mathrm{JH}$, was found to bind to dFoxo and inhibits the transcription of several of its targets, including $I n r^{26}$. Altogether, our data indicate that 
a
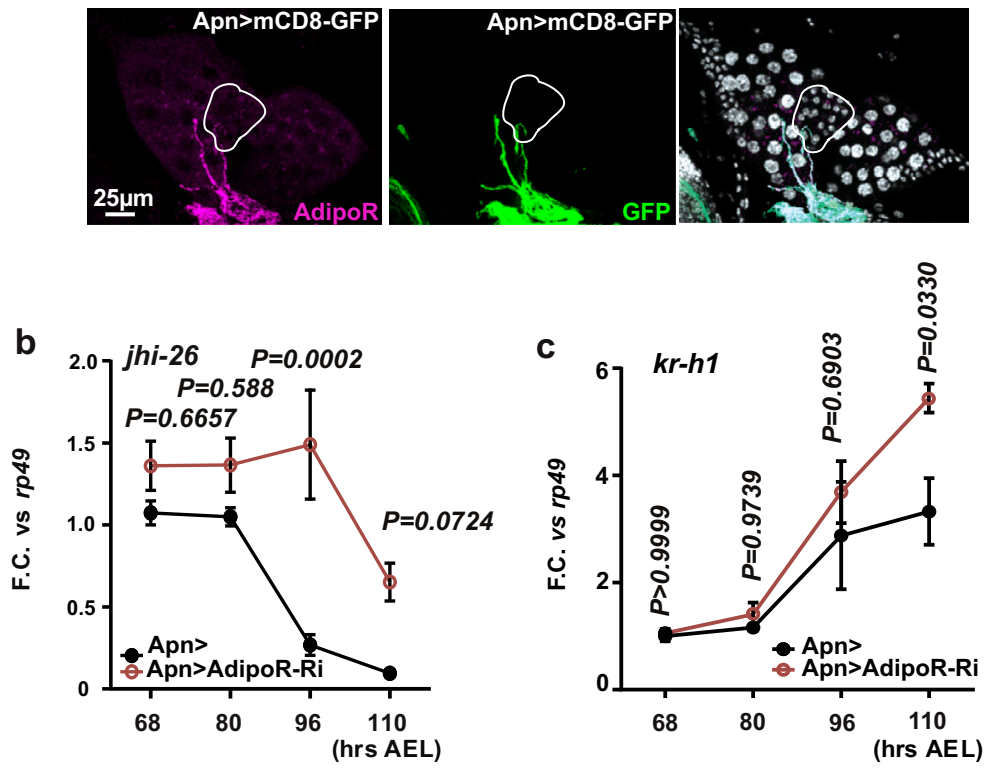

d

e
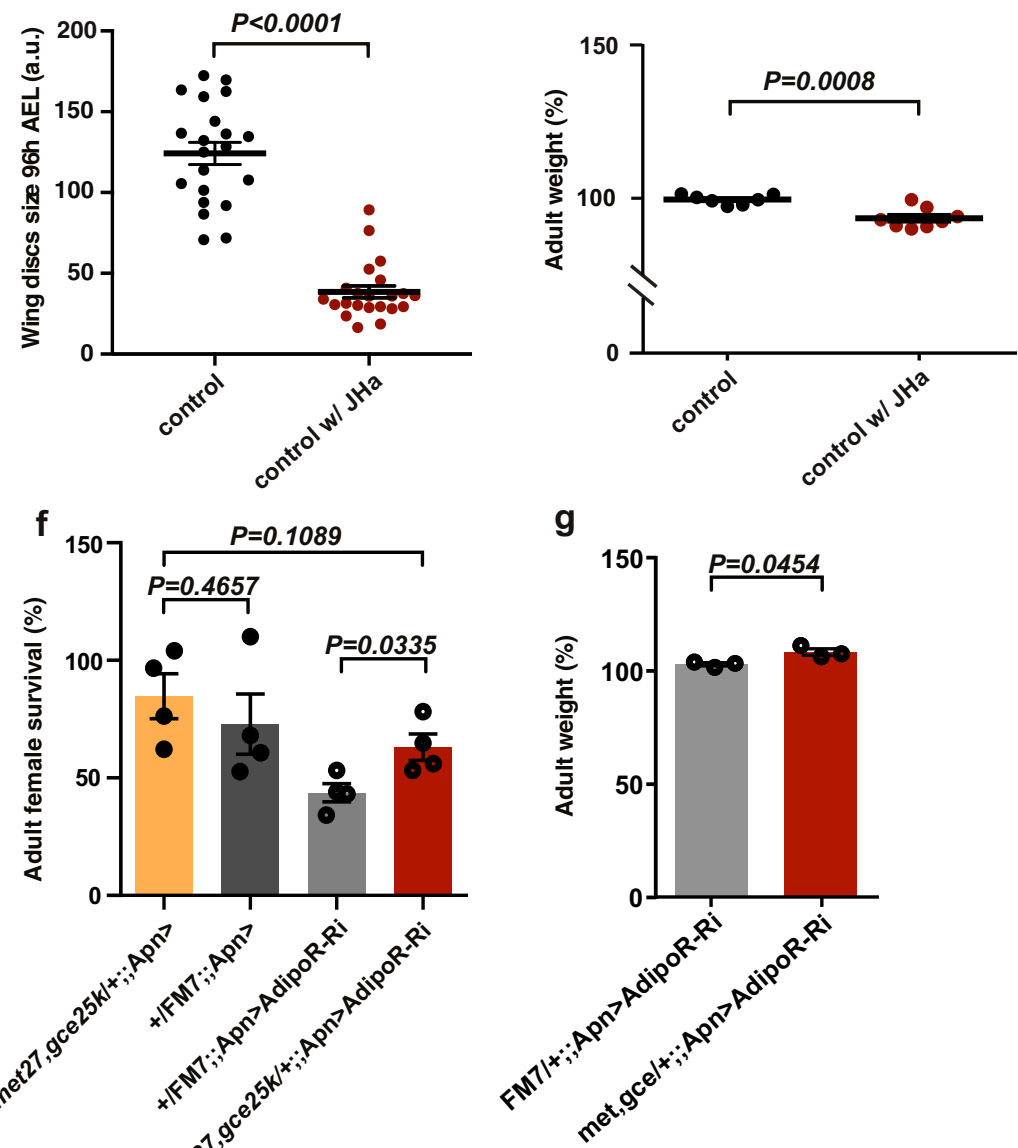

inhibition of adiponectin receptor signaling in the brain induces peripheral insulin resistance through an increase in $\mathrm{JH}$ signaling.

Finally, perturbations of grp78 expression in fat cells (lpp> grp78-Ri and $l p p>g r p 78)$ sensibly modified the levels of circulating Dilp2 (Fig. 4h, i), indicating that Grp78 acts as an adipose antagonist of AdipoR function and participates in peripheral insulin resistance in HSD conditions.

In conclusion, we describe a brain circuitry controlling peripheral insulin response, whereby a limited number of AdipoR-positive neurons regulate peripheral $\mathrm{JH}$ signaling, which in turn inhibit insulin response in peripheral organs. Moreover, we identify Grp78, a secreted protein previously identified in UPR response, as an antagonistic interactor of dAdipoR and its possible agonist ligands, produced upon high sugar feeding and 
Fig. 3 Central AdipoR function controls JH signaling. a Anti-AdipoR and anti-GFP immunostaining of Apn > mCD8-GFP larval brains. Brain and ring gland are stained with DAPI, the corpora allata are circled. ( $n=18$ in five independent experiments). $\mathbf{b}, \mathbf{c}$ jhi-26 and kr-h1 expression on whole larvae Apn > AdipoR-Ri (red) and Apn > (black). qRT-PCR profiles, fold changes (FC) normalized to rp49. Statistical significance was tested using ordinary two-way ANOVA with Sidak's multiple comparisons test. $n=2-3$ biologically independent replicates per time point (three independent experiments). $\mathbf{d}$ Measure of $\mathrm{JH}$ analog (pyroproxyfen)-feeding wing disc size. (control $n=22$, black; control w/Jha $n=21$, red, in three independent experiments). Statistical significance was tested using ordinary two-way ANOVA with Sidak's multiple comparisons test. e Measure of adult weight in JH analog feeding (control $n=7$, black; control w/Jha $n=8$, red). Statistical significance was tested using a two-sided unpaired $t$-test with Welch's correction. $\mathbf{f}$, $\mathbf{g}$ Rescue of Apn > AdipoR-Ri female lethality (f) and adult weight (g) by met27, gce25k heterozygous mutations (met27, gce25k/FM7; Apn > AdipoR-Ri in red compared to $A p n>A$ dipo $R-R i$ in light gray). $n=4$ independent experiments (in $\mathbf{f}$ ), $n=3$ groups of 15 females of each genotype (in $\mathbf{g}$ ). Statistical significance was tested using two-sided unpaired $t$-test with Welch's correction. Data were presented as mean values \pm SEM. $P$ values are indicated in all panels. a.u. arbitrary units. Source data are provided as a Source Data file.

participating in peripheral insulin resistance (Supplementary Fig. 4c). Some of the properties of JH in controlling developmental processes are shared with the vertebrate thyroid hormones ${ }^{27}$. Hyperthyroidism is associated with peripheral insulin resistance, suggesting mechanistic conservation of their metabolic functions. Finally, Grp78 is found in the blood of mice fed an HSD and heterozygous grp78 mutant mice are protected from obesity when fed a hypercaloric diet ${ }^{28}$, suggesting that central adiponectin signaling and Grp78 could play conserved roles in maintaining energy homeostasis in vertebrates.

\section{Methods}

Fly stocks and food. The following Gal4 lines were used in this study: Apn-Gal4 (Janelia \#48522, corresponding to a subregion of the promoter of the Gycbeta100B gene, referred to as Apn-Gal4 in the text); dilp2-Gal4 (BDSC\#37516), MHC-Gal4 (BDSC\#55133), ptc-Gal4 (BDSC\#2017), lpp-Gal429, Akh-Gal4 (BDSC\#25683), and elav-Gal4 (BDSC\#458). To invalidate AdipoR, two independent UAS-RNAi and a lethal EP insertion in the AdipoR gene were used: UAS-AdipoR-RNAiGD (called UAS-AdipoR-Ri in this study; VDRC\#v40936), UAS-AdipoR-RNAiTRIP (called UAS-AdipoR-TRIP in this study; BDSC\#67814), and AdipoR-G6641 (called Adi$p o R^{M}$ in this study; BDSC\#31800). Other lines used: white1118 (control w); UAS$m C D 8 G F P$ (BDSC\#5137), CaLexA system (lexAop-CD8-GFP-2A-CD8-GFP; UASmLexA-VP16-NFAT, LexAop-rCD2-GFP) ${ }^{11} ; m^{27} t^{27}, g c e^{25 k}$ (gift from I. MiguelAliagua);UAS-gpr78-RNAi (VDRC\#v101766); UAS-grp78 (BDSC\#5843), UAS$s G F P^{30}$, tGPH (BDSC\#8163). Lines used for circulating Dilp2 measurements were: Dilp2HF insertions combined or not with elav-Gal4, lpp-Gal4, dilp2-Gal4, or ApnGal4 (original and combined $g d 2 H F$ lines from refs. ${ }^{31,32}$ and this study). UAS-gfpRNAi (BDSC\#9330) was used as a control for all the crosses and experiments (unless if notified).

Animals were reared on reference food (normal diet, ND) at $25^{\circ} \mathrm{C}$ (or $29^{\circ} \mathrm{C}$ ) containing per liter: $10 \mathrm{~g}$ agar, $82,5 \mathrm{~g}$ corn flour, $60 \mathrm{~g}$ sugar, $34 \mathrm{~g}$ inactivated yeast extract, and $375 \mathrm{~g}$ Moldex (in Ethanol). LSD corresponds to the same without sugar and HSD to the same with $240 \mathrm{~g}$ sugar.

Animal volume and weight measurements. Flies were allowed to lay eggs $4 \mathrm{~h}$ on agar plates with yeast paste and first instar larvae were synchronized and transferred on the desired food under control conditions ( $30 \mathrm{~L} 1 /$ vial). Animals were allowed to develop until the stage of interest at $29^{\circ} \mathrm{C}$. Developmental timing, animal volume, adult weight, and larval imaginal disks/adult wing sizes were performed as described previously ${ }^{33,34}$.

Determination of larval and pupal volume. Flies were allowed to lay eggs and first instar larvae were synchronized and transferred on the desired food under control conditions ( $30 \mathrm{~L} 1 / \mathrm{vial}$ ). Animals were allowed to develop until pupal stage at $29^{\circ} \mathrm{C}$. Pupal volume was measured using Fiji and calculated by using the formula: $(4 / 3) \pi(\mathrm{L} / 2)(1 / 2) 2$ (L length; 1 diameter).

Plasmids and transgenic flies. To generate transgenic UAS- $h A d i p o Q$ (Human Adiponectin), Adiponectin human cDNA (Origene, \#TP315161) was PCR amplified and subcloned, using the Gateway cloning system (Invitrogen \#K-2400-20), in Flag-tagged-UAS as destination vector (Carnegie Institution of Washington Vector collection). UAS-AdipoR-Myc was obtained from cloning a synthetic AdipoR cDNA (GeneSynthesis; GenScript) into a Myc-tagged-UAS as destination vector (Carnegie Institution of Washington Vector collection), using the Gateway cloning system (Invitrogen). A sequence of AdipoR corresponding to the cytoplasmic part of the receptor only (amino acids 1-201) was PCR amplified and subcloned in Myctagged-UAS as destination vector (Carnegie Institution of Washington Vector collection) to generate an activated form of AdipoR (AdipoR-act). grp78 cDNA was subcloned from FlyORF clone (DGRC \#GEO03341) to an HA-tagged-UAS vector as destination vector using the Gateway cloning system (Invitrogen; Carnegie
Institution of Washington Vector collection). Expression of the transgenes was validated by transfection in S2 cells and western blot. Constructs were then introduced into germ line by injections in the presence of the integrase (BestGene). (Primers sequences provided in Table S2; material available upon request).

Adiponectin, JHa, and $20 \mathrm{E}$ feeding. Human adiponectin recombinant protein (Merck, \#SRP4901) was added to the food at a $5 \mathrm{ug} / \mathrm{ml}$ final concentration. Pyriproxifen (PESTANAL ${ }^{\circledR}$, Merck, \#34174) was used as JH analog (JHa, Ethanol stock at $1 \mathrm{mg} / \mathrm{ml}$ ) at $2 \mathrm{ppm}$ final in food used to reared animals (compared to control food without JHa). 20E (Sigma Aldrich, \#5289-74-7) was added at $0.75 \mathrm{mg} / \mathrm{ml}$ to yeast paste during larval third instar on synchronized animals ${ }^{35}$. Dilution in PBS of a stock solution of $20 \mathrm{E}$ at $5 \mathrm{mg} / \mathrm{ml}$ in ethanol was used and ethanol was used as a control.

Antibodies and immunohistochemistry. To generate antisera against AdipoR protein (available upon request), two different couples of peptides were used as an immunogen in rats (doubleX program; Eurogentec). The chosen peptides correspond to amino acids KRRGWGPEDSLSPNDL (75-90)/LWDKFSEPALRPLRAG (321-334) and LQDNDFLHRGHRPPL (173-187)/VPERWFPGKFDIWGQ (388-402) of the sequence. Both are giving the same pattern. Wandering larvae were dissected in cold PBS on ice, fixed in 37\% methanol-free formaldehyde (Polysciences Inc., \#18814-10) for $25 \mathrm{~min}$, washed several times in PBS $+0.1 \%$ Triton X-100 (PBT) before $2 \mathrm{~h}$ blocking in PBT containing 10\% BSA. Primary antibodies were incubated overnight at $4{ }^{\circ} \mathrm{C}$. The following primary antibodies were used: rat anti-AdipoR (1:400, this study); chicken anti-GFP (1:10000, Abcam \#mAb13970; RRID:AB 300798), rabbit anti-Dilp5 (1:500, generated in Leopold's lab $)^{36}$, rabbit anti-Akh $(1 / 500)^{37}$, guinea pig anti-PTTH $(1: 400$, generated in Leopold's lab) ${ }^{38}$. Alexa conjugated secondary antibodies were incubated for $2 \mathrm{~h}$ at room temperature then tissues of interest were mounted, after extensive washes, in SlowFade Gold antifade reagent (Molecular Probes, \#S36936). The following secondary antibodies were used (1:400; Thermo Fisher Scientific): Alexa Fluor 488 goat anti-chicken (\#A-11039), Alexa Fluor 546 goat anti-rat (\#A-11081), Alexa Fluor 488 goat anti-rabbit (\#A-11008), and Alexa Fluor 488 goat anti-guinea pig (\#A-11073).

Fluorescence images were acquired by sequential scanning using a Leica SP5 DS (20x and $40 \times$ objectives) and processed with Fiji ${ }^{39}$ Software.

Antibody staining quantification. For quantification of AdipoR staining in the APNs, stacks were taken for each cluster of Apn in each lobe from top to bottom limit of the staining. Slices of the whole stack were used to evaluate the fluorescence intensity using Fiji ${ }^{39}$ (StagReg, Time Series Analyser V3.0, and Roi Manager plugins).

NFAT quantification. We used the calcium reporter UAS-CaLexA (calciumdependent nuclear import of LexA, lexAop-CD8-GFP-2A-CD8-GFP; UAS-mLexAVP16-NFAT, and LexAop-rCD2-GFP), allowing $\mathrm{Ca}^{++}{ }^{+-d e p e n d e n t ~ a c t i v a t i o n ~ o f ~ G F P ~}$ transcription $^{11}$. For sugar tests, brains from CalexA; Apn $>$ or CaLexA; Apn >, $>$ AdipoR-Ri pre-wandering larvae (still in the food), grown on 0xS-1xS-4xS (LSDND-HSD) foods, were dissected on ice in PBS, and immunostaining was performed as described above. For hAdipoQ feeding, CalexA; Apn $>$ animals grew on $4 \mathrm{xS}$ food supplemented with $5 \mathrm{ug} / \mathrm{ml}$ of recombinant human adiponectin (Merck, \#SRP4901) were incubated overnight on a petri dish. Chicken anti-GFP antibody $(1: 10,000$, Abcam \#mAb13970) was used as primary.

For quantification, stacks were taken for each cluster of Apn in each lobe from top to bottom limit of the staining. Slices of the whole stack were used to evaluate the fluorescence intensity using Fiji ${ }^{39}$ (StagReg, Time Series Analyser V3.0, and Roi Manager plugins). For each brain, one cluster was taken into account and three to four individual neurons per cluster were measured.

Ex-vivo incubation. Fat bodies from tGPH larvae were incubated with the desired compound or with control solutions (ethanol or water) diluted in HL6 medium ${ }^{40}$, at room temperature: $\mathrm{JHa}(2 \mathrm{ppm}$ for $30 \mathrm{~min}$; Merck, \#34174) or Insulin (0.5 uM 

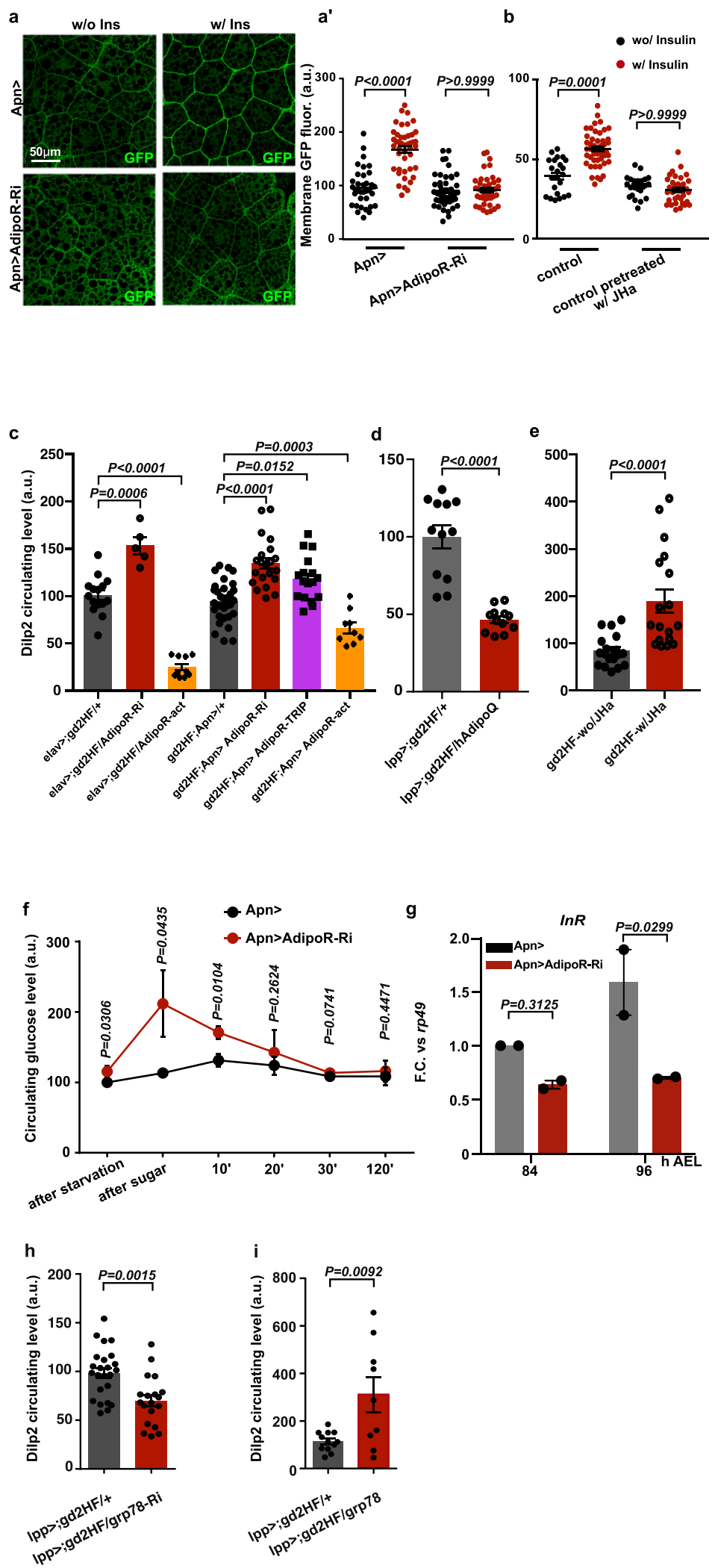

for $20 \mathrm{~min}$; Merck, \#i9278). For sequential incubations, after $30 \mathrm{~min}$ of incubation with $\mathrm{JHa}$ (or EtOH as a control), insulin (or $\mathrm{H} 2 \mathrm{O}$, as a control) was added for $30 \mathrm{~min}$. The incubation mix was then removed and tissues were fixed and processed as described above. Chicken anti-GFP antibody (1:10,000, Abcam \#mAb13970) was used as primary. Images were obtained with Leica SP5 (objective $\times 40)$ and processed with Fiji Software ${ }^{39}$. For quantification of fluorescence, an average of independent measurements per fat bodies of the indicated genotypes was performed.
Brains from Apn $>$ CalexA larvae grown in 0xS were dissected in Schneider medium (Gibco $\left.{ }^{\mathrm{TM}} \# 21020024\right)$ with FBS and incubated overnight at $18^{\circ} \mathrm{C}$ with $20 \mathrm{ul}$ of the hemolymph form control $(l p p>$ ) or test (lpp > grp78-Ri) larvae grown in $0 \times \mathrm{S}$ or $4 \times \mathrm{S}$. Tissues were then fixed and immunostaining was processed as described above with chicken anti-GFP antibody (see previously).

S2 cells transfection and co-immunoprecipitation. UAS-AdipoR-Myc, UAShAdipoQ-Flag, and/or UAS-grp78-HA were transfected in naive S2 cells with the 
Fig. 4 Central AdipoR function and JH signaling control peripheral insulin sensitivity. a GFP localization at the membrane in $t G P H$ fat body explants $-/+$ incubation with human insulin, as a marker for insulin response. Fat bodies are stained with anti-GFP. (minimum $n=8$ in two independent experiments). a Quantification of membrane GFP staining in individual cells of larval fat bodies explants wo/insulin (black) or w/insulin incubation (red) from (a) Apn $>$ wo/ins $n=36$, w/ins $n=44 ;$ Apn $>$ AdipoR-Ri wo/ins $n=44$, w/ins $n=44$ ). b Quantification of membrane GFP staining in individual cells of $t G P H$ larval fat bodies explants pretreated or not with JHa before incubation with insulin (wo/insulin, black; w/insulin, red) (relates to Fig. S4c) (control wo/ins $n=23$, w/ins $n=47$; control wo/ins $n=23$, w/ins $n=37)$. $\ln \left(\mathbf{a}^{\prime}, \mathbf{b}\right) n=$ number of averaged GFP intensity in fat bodies pieces. Statistical significance was tested using ordinary one-way ANOVA (Kruskal-Wallis test) with Dunn's multiple comparisons test. c-e Measure of hemolymph Dilp2 by ELISA in elav > ; gd2HF/+ ( $n=15$, gray), elav > ;gd2HF/AdipoR-Ri $(n=5$, red), elav > ;gd2HF/AdipoR-act $(n=10$, orange) in two independent experiments; gd2HF;Apn > /+ ( $n=32$, gray), gd2HF;Apn >/AdipoR-Ri ( $n=21$, red; four independent experiments), gd2HF;Apn >/AdipoR-TRIP ( $n=17$, purple; two independent experiments), and $g d 2 H F ; A p n>/ A d i p o R$-act $(n=9$, orange; three independent experiments); in lpp > ;gd2HF ( $n=12$, black) and lpp > ;gd2HF/ $h \operatorname{AdipoQ}(n=12$, red) in two independent experiments (in c, d), and upon feeding larvae with $\mathrm{JHa}$ during development $g \mathrm{~d} 2 \mathrm{HF}$-wo/Jha $(n=18$, black) and $g d 2 \mathrm{HF}-\mathrm{W} / \mathrm{Jha}(n=18$, red) in three independent experiments (in $\mathbf{e}) . n=$ number of independent measurements. Statistical significance was tested using ordinary two-way ANOVA with Sidak's multiple comparisons test. $\mathbf{f}$ Larval glucose clearance after glucose feeding. Statistical significance at each time point was tested using an unpaired two-sided $t$-test. $n=3$ independent experiments. $\mathbf{g}$ InR expression on whole larvae (qRT-PCR profiles, fold changes (FC) normalized to rp49). Statistical significance was tested using ordinary two-way ANOVA with Sidak's multiple comparisons test. $n=3$ biologically independent replicates per experiment (two independent experiments). h, i Measure of hemolymph Dilp2 by ELISA in lpp > ;gd2HF/grp78-Ri ( $n=19$, red) and $l p p>$; $g d 2 \mathrm{HF} / g r p 78(n=9$, red) compared to $I p p>; g d 2 H F(n=24$ and $n=11$, respectively, black) in four and three independent experiments (h and $\mathbf{i}$, respectively) $n=$ number of independent averaged measurements. Statistical significance was tested using ordinary two-way ANOVA with Sidak's multiple comparisons test. Data were presented as mean values \pm SEM. $P$ values are indicated in all panels. a.u. arbitrary units. Source data are provided as a Source Data file.

pAct-GAL4 vector using Lipofectamine reagent kit (Invitrogen \#18324012) in a proportion of 1:1:1. Lysis and immunoprecipitation were performed as described previously 41

Western blot. Hemolymph samples from $96 \mathrm{~h}$ AEL larvae of the indicated genotypes, reared on $0 \times \mathrm{S}$ (LSD) and $4 \times \mathrm{S}$ (HSD) foods, were extracted as described in ref. ${ }^{42}$, and prepared for western blotting. For control ( $l p p>$ GFP-RNAi; Fig. 2a) and sGFP expressing larvae (lpp $>s G F P$, Fig. S2d), the experiment was performed on 10 ug of proteins. For $l p p>g r p 78-R N A i$, the experiment was performed on $5 \mathrm{ul}$ of pure larval hemolymph, the low amount of total serum proteins preventing the measurement of protein amount by Bradford. In both cases, cv-d was used as a normalizer for hemolymph proteins ${ }^{43}$.

Proteins (from IP and hemolymph) were resolved by SDS-PAGE, using $4-12 \%$ gradient gels (NuPage Novex Gel, Invitrogen \#NP0335) ${ }^{41}$. Primary antibodies used were: rabbit anti-Grp78 (1:1000, Novus Biologicals \#NBP1-06274), rabbit AntiGFP N-ter (1:1000, Merck, \#G1544), guinea-pig anti-Cv-d $\left(1: 2000^{43}\right.$, gift from Eaton lab); mouse anti-Myc 9E10 (1:1000, Santa Cruz Biotechnology, \#sc-40), rabbit anti-Myc A-14 (1:1000, Santa Cruz Biotechnology, \#sc-789), rat anti-HA (1:1000, Merck/Roche, \#ROAHAHA), and mouse anti-FLAG ${ }^{\oplus}$ M2 (1:1000, Merck, F3165). Membranes were washed in PBS tween $0.1 \%$ and incubated with secondary antibodies in this buffer for $2 \mathrm{~h}$ at room temperature. The following HRP conjugated secondary antibodies were used (Thermo Fisher Scientific): Goat antiRabbit (1:2500, \#G-21234), Goat anti-mouse (1:2500, \#G-21040), and anti-guinea pig (1:2500, \#A18769). Chemiluminescence was observed using the ECL detection system (Pierce). Images were generated using Fiji ${ }^{39}$.

Glucose tolerance measurements. Seventy-two hours AED larvae were starved for $2 \mathrm{~h}$ on a humid petri dish plated with agarose and immerged in a petri dish containing a saturated sugar solution for $45 \mathrm{~min}$. Larvae are removed from sugar, washed, dried, and put back on a humid petri dish for a challenging time. For each point, hemolymph of three times eight larvae (in triplicates/genotype) was picked on ice and submitted to total glucose measurement as described in ref. ${ }^{44}$, using the GAGO-20 kit from Merck (according to the manufacturer's instructions). Absorbance at $540 \mathrm{~nm}$ was measured using a TECAN microplate reader.

Dilp2HF Elisa. Circulating Dilp2 was quantified by sandwich Elisa as described in ref. ${ }^{31}$. Almost three times 1 ul of clean hemolymph was picked from groups of ten larvae of the indicated genotypes (one biological replicate) or fed with $2 \mathrm{ppm} \mathrm{JHa}$ during all development and submitted, to Elisa test. The relative ratio of tested animals is given relative to control animals, normalized to the volume of hemolymph.

Ecdysone measurements. Five Larvae were homogenized and extracted in $250 \mathrm{ul}$ methanol or $5 \mathrm{ul}$ of hemolymph in $200 \mathrm{ul}$ methanol. Extractions were then submitted to a competitive ELISA test as previously published ${ }^{45}$ to evaluate the amount of total or circulating 20E, using the detection kit from SpiBio (Bertin reagents \#A05120) as recommended by the manufacturer. Absorbance at $415 \mathrm{~nm}$ was detected using a TECAN microplate reader.
qRT-PCR. Whole larvae (all figures panels except Fig. S4a) or dissected tissues (Fig S4a) were collected, washed in PBS $1 \times$, and dried, then frozen in liquid nitrogen Total RNA was extracted using Qiagen RNeasy lipid tissue mini kit according to the manufacturer protocol (\#74804). RNA samples ( $3 \mu \mathrm{g}$ per reaction) were treated with DNase and reverse-transcribed using SuperScript II reverse transcriptase (Invitrogen \#18064014) and the generated cDNA was used for real-time RT-PCR (StepOne Plus, Applied Biosystem) using PowerSYBRGreen PCR master mix (Applied Biosystem \#4367659) as previously described ${ }^{46}$. Three biological replicates were collected for each sample and technical triplicate was conducted for each (Primers sequences are provided in Table S2).

DTT and tunicamycin treatment. Control larvae were allowed to grow either on LSD or HSD diet until pre-wandering. For DTT treatment, three groups of ten LSD-fed larvae were starved $2 \mathrm{~h}$ and incubated for $4 \mathrm{~h}$ in Schneider's medium \pm 5 mM DTT (Merck \#10197777001). Larvae were then washed and frozen in liquid nitrogen. Three groups of ten LSD- or HSD-fed larvae were washed with PBS and frozen simultaneously. RNA extraction and qRT-PCR were performed as described above. Independent experiments were performed using three biological replicates tested in triplicates. For tunicamycin treatment, fat tissues from $l p p>$ $x b p 1-G F P$ larvae were incubated $1 \mathrm{~h}$ either with $10 \mathrm{ug} / \mathrm{ml}$ tunicamycin $(1: 1000$ dilution of $10 \mathrm{mg} / \mathrm{ml}$ stock solution, Sigma Aldrich \#T7765) or with 1:1000 DMSO in Schneider's medium at room temperature. Tissues were then washed with PBS and fixed in 37\% methanol-free formaldehyde (Thermo Fisher Scientific, \#28906). Anti-GFP immunostaining was performed as described above.

Mass spectrometry analysis. Ubiquitously expressed Myc-AdipoR protein was immunoprecipitated from larvae using magnetic microparticles (MACS purification system, Miltenyi Biotech) according to the manufacturer's instructions and as previously described ${ }^{47}$. $\mu$ MACS magnetic microbeads are coated with a monoclonal anti-Myc antibody (Miltenyi Biotec). Negative controls, wild-type larvae, and the negative control protein myc-SMO (kindly provided by Dr L. Ruel) were purified by affinity with the same conditions to remove unspecific proteins. A positive control was carried out with a co-expression of Myc-AdipoR with hAdipoQ. Co-immunoprecipitation experiments were carried out in triplicates. Proteins were eluted out of the magnetic stand with the SDS-loading buffer from the kit.

Eluted proteins were digested with sequencing-grade trypsin (Promega) and analyzed by nanoLC-MS/MS on a QExactive+ mass spectrometer coupled to an EASY-nanoLC-1000 (Thermo Fisher Scientific) as described previously ${ }^{48}$. Data were searched against the Flybase database (release r6.16) with a decoy strategy. Peptides were identified with Mascot algorithm (version 2.5, Matrix Science, London, UK) and data were further imported into Proline 1.4 software (http:// proline.profiproteomics.fr/). Proteins were validated on Mascot pretty rank equal to 1 , and $1 \%$ FDR on both peptide spectrum matches (PSM score) and protein sets (protein set score). The total number of MS/MS fragmentation spectra was used to quantify each protein from at least three independent biological replicates. This spectral count was submitted to a negative-binomial test using an edgeR GLM regression through $\mathrm{R}$ ( $\mathrm{R}$ v3.2.5). For each identified protein, an adjusted $P$ value corrected by Benjamini-Hochberg was calculated, as well as a protein fold change (FC). We used a in-house developped R script, called IPinquiry, freely available under Github (https://github.com/hzuber67/IPinquiry4). 
RNAi screen of candidate genes. Candidate genes were selected on the enrichment value of the MS results. A blind RNAi screen was performed using two independent lines for each candidate (VDRC KK/GD and TRIP, if available). The RNAi were expressed with $p p l>$ (expressed in the gut and the fat body, both tissues assumed to be the two most important secretory tissues of the larva). Pupal lethality was checked as a read-out.

Top candidate genes of the MS were screened in priority and among them, ones known to encode secreted or putative secreted proteins. A non-exhaustive list of candidates is presented in Fig. S2c.

Statistical analysis and reproducibility. Statistical analyses were performed with Graphpad Prism V9. The statistical test used for each experiment and the $P$ values are indicated in the corresponding Figure and Figure legends. Experiment replications are summarized in Table S1.

Software. Mascot Algorithm v2.5 (Matrix Science, London, UK) and Proline software v1.4 (http://proline.profiproteomics.fr) were used for the mass spectrometry.

GraphPad Prism V9 was used for statistical analysis and graphical representation of the data. Fiji (ImageJ version 2.0.0-RC-69/1.52n) was used for Image treatment from microscopy and western blot. Affinity Photo v.1.8.5 and Affinity Designer v.1.9.3 (Serif, Europe) were used for Figures elaboration.

\section{Data availability}

The processed mass spectrometry data are available at PRIDE repository:

ProteomeXchange accession: PXD013689

Project Webpage: http://www.ebi.ac.uk/pride/archive/projects/PXD013689

FTP Download: ftp://ftp.pride.ebi.ac.uk/pride/data/archive/2021/06/PXD013689/

Source data are provided with this paper.

\section{Code availability}

The custom R script used for mass spectrometry analysis is called IPinquiry and is freely available from Github (https://github.com/hzuber67/IPinquiry4).

Received: 10 December 2020; Accepted: 16 July 2021;

Published online: 24 September 2021

\section{References}

1. Timper, K. \& Brüning, J. C. Hypothalamic circuits regulating appetite and energy homeostasis: pathways to obesity. Dis. Model. Mech. 10, 679-689 (2017).

2. Pozo, M. \& Claret, M. Hypothalamic control of systemic glucose homeostasis: the pancreas connection. Trends Endocrinol. Metab. 29, 581-594 (2018).

3. Arita, Y. et al. Paradoxical decrease of an adipose-specific protein, adiponectin, in obesity. Biochem. Biophys. Res. Commun. 257, 79-83 (1999).

4. Tsuchida, A. et al. Insulin/Foxol pathway regulates expression levels of adiponectin receptors and adiponectin sensitivity. J. Biol. Chem. 279, 30817-30822 (2004)

5. Maeda, N. et al. Diet-induced insulin resistance in mice lacking adiponectin/ ACRP30. Nat. Med. 8, 731-737 (2002).

6. Qi, Y. et al. Adiponectin acts in the brain to decrease body weight. Nat. Med. 10, 524-529 (2004).

7. Kubota, N. et al. Adiponectin stimulates AMP-activated protein kinase in the hypothalamus and increases food intake. Cell Metab. 6, 55 (2007).

8. Kos, K. et al. Adiponectin and resistin in human cerebrospinal fluid and expression of adiponectin receptors in the human hypothalamus. J. Clin. Endocrinol. Metab. 92, 1129-1136 (2007).

9. Kwak, S.-J. et al. Drosophila adiponectin receptor in insulin producing cells regulates glucose and lipid metabolism by controlling insulin secretion. PLoS ONE 8, e68641 (2013).

10. Yamauchi, T. et al. Cloning of adiponectin receptors that mediate antidiabetic metabolic effects. Nature 423, 762 (2003).

11. Masuyama, K., Zhang, Y., Rao, Y. \& Wang, J. W. Mapping neural circuits with activity-dependent nuclear import of a transcription factor. J. Neurogenet. 26, 89-102 (2012)

12. Niccoli, T. et al. Increased glucose transport into neurons rescues Ab toxicity in Drosophila. Curr. Biol. 26, 2291-2300 (2016).

13. Mote, P. L., Tillman, J. B. \& Spindler, S. R. Glucose regulation of GRP78 gene expression. Mech. Ageing Dev. 104, 149-158 (1998).

14. Zhang, Y., Liu, R., Ni, M., Gill, P. \& Lee, A. S. Cell surface relocalization of the endoplasmic reticulum chaperone and unfolded protein response regulator GRP78/BiP. J. Biol. Chem. 285, 15065-15075 (2010).
15. Hetz, C. \& Mollereau, B. Disturbance of endoplasmic reticulum proteostasis in neurodegenerative diseases. Nat. Rev. Neurosci. https://doi.org/10.1038/ nrn3689 (2014).

16. Dubrovsky, E. B., Dubrovskaya, V. A., Bilderback, A. L. \& Berger, E. M. The isolation of two juvenile hormone-inducible genes in Drosophila melanogaster. Dev. Biol. 224, 486-495 (2000).

17. Riddiford, L. M. \& Ashburner, M. Effects of juvenile hormone mimics on larval development and metamorphosis of Drosophila melanogaster. Gereral Comp. Endocrinol. 82, 172-183 (1990).

18. Abdou, M. A. et al. Drosophila Met and Gce are partially redundant in transducing juvenile hormone action. Insect Biochem. Mol. Biol. 41, 938-945 (2011).

19. McBrayer, Z. et al. Prothoracicotropic hormone regulates developmental triming and body size in Drosophila. Dev. Cell 13, 857-871 (2007).

20. Shimell, M. J. et al. Prothoracicotropic hormone modulates environmental adaptive plasticity through the control of developmental timing. Development 145, dev159699 (2018).

21. Britton, J. S., Lockwood, W. K., Li, L., Cohen, S. M. \& Edgar, B. A. Drosophila's insulin/PI3-kinase pathway coordinates cellular metabolism with nutritional conditions. Dev. Cell 2, 239-249 (2002).

22. Musselman, L. P. et al. A high-sugar diet produces obesity and insulin resistance in wild-type Drosophila. Dis. Model Mech. 4, 842-849 (2011).

23. Pasco, M. Y. \& Léopold, P. High sugar-induced insulin resistance in Drosophila relies on the Lipocalin Neural Lazarillo. PLoS ONE 7, e36583 (2012).

24. Haselton, A. et al. Partial ablation of adult drosophila insulin-producing neurons modulates glucose homeostasis and extends life span without insulin resistance. Cell Cycle 9, 3063-3071 (2010).

25. Patti, M. E. \& Kahn, C. R. Lessons from transgenic and knockout animals about noninsulin-dependent diabetes mellitus. Trends Endocrinol. Metab. 7, 311-319 (1996).

26. Kang, P. et al. Drosophila Kruppel homolog 1 represses lipolysis through interaction with dFOXO. Sci. Rep. 7, 16369 (2017).

27. Flatt, T., Moroz, L. L., Tatar, M. \& Heyland, A. Comparing thyroid and insect hormone signaling. Integr. Comp. Biol. 46, 777-794 (2006).

28. Ye, R. et al. Grp78 heterozygosity promotes adaptive unfolded protein response and attenuates diet-induced obesity and insulin resistance. Diabetes 59, 6-16 (2010)

29. Brankatschk, M. \& Eaton, S. Lipoprotein particles cross the blood-brain barrier in Drosophila. J. Neurosci. 30, 10441-10447 (2010).

30. Entchev, E. V., Schwabedissen, A. \& González-Gaitán, M. Gradient formation of the TGF- $\beta$ homolog Dpp. Cell 103, 981-992 (2000).

31. Park, S. et al. A genetic strategy to measure circulating drosophila insulin reveals genes regulating insulin production and secretion. PLoS Genet. 10, e1004555 (2014).

32. Meschi, E., Léopold, P. \& Delanoue, R. An EGF-responsive neural circuit couples insulin secretion with nutrition in Drosophila. Dev. Cell 48, 76-86 (2019). e5.

33. Agrawal, N. et al. The Drosophila TNF Eiger is an adipokine that acts on insulin-producing cells to mediate nutrient response. Cell Metab. 23, 675-684 (2016).

34. Colombani, J. et al. Drosophila Lgr3 couples organ growth with maturation and ensures developmental stability. Curr. Biol. 25, 2723-2729 (2015).

35. Delanoue, R., Slaidina, M. \& Léopold, P. The steroid hormone ecdysone controls systemic growth by repressing dMyc function in drosophila fat cells. Dev. Cell 18, 1012-1021 (2010).

36. Géminard, C., Rulifson, E. J. \& Léopold, P. Remote control of insulin secretion by fat cells in Drosophila. Cell Metab. 10, 199-207 (2009).

37. Isabel, G., Martin, J. R., Chidami, S., Veenstra, J. A. \& Rosay, P. AKHproducing neuroendocrine cell ablation decreases trehalose and induces behavioral changes in Drosophila. Am. J. Physiol. Regul. Integr. Comp. Physiol. 288, R531-R538 (2005).

38. Yamanaka, N. et al. Neuroendocrine control of Drosophila larval light preference. Science 341, 1113-1116 (2013).

39. Schindelin, J. et al. Fiji: an open-source platform for biological-image analysis. Nat. Methods 9, 676-682 (2012).

40. Bjordal, M., Arquier, N., Kniazeff, J., Pin, J. P. \& Léopold, P. Sensing of amino acids in a dopaminergic circuitry promotes rejection of an incomplete diet in drosophila. Cell 156, 510-521 (2014).

41. Andersen, D. S. et al. The Drosophila TNF receptor Grindelwald couples loss of cell polarity and neoplastic growth. Nature 522, 482-486 (2015).

42. Rodenfels, J. et al. Production of systemically circulating Hedgehog by the intestine couples nutrition to growth and development. Genes Dev. 28, 2636-2651 (2014)

43. Palm, W. et al. Lipoproteins in Drosophila melanogaster-assembly, function, and influence on tissue lipid composition. PLoS Genet. 8, e1002828 (2012)

44. Tennessen, J. M., Barry, W. E., Cox, J. \& Thummel, C. S. Methods for studying metabolism in Drosophila. Methods 68, 105-115 (2014). 
45. Rewitz, K. F., Yamanaka, N. \& O’Connor, M. B. Steroid hormone inactivation is required during the juvenile-adult transition in Drosophila. Dev. Cell 19, 895-902 (2010).

46. Delanoue, R. et al. Drosophila insulin release is triggered by adipose stunted ligand to brain methuselah receptor. Science 353, 1553-1556 (2016).

47. Stoetzel, C. et al. A mutation in VPS15 (PIK3R4) causes a ciliopathy and affects IFT20 release from the cis-Golgi. Nat. Commun. 7, 13586 (2016).

48. Chicher, J. et al. Purification of mRNA-programmed translation initiation complexes suitable for mass spectrometry analysis. Proteomics 15, 2417-2425 (2015).

\section{Acknowledgements}

We thank T. Pihl for help with the experiments and laboratory members for discussions; We thank I. Miguel-Aliaga, K. Masuyama, the Bloomington Stock Center, and the Vienna Drosophila RNAi Center for fly stocks. Funding is from Inserm, CNRS, the Fondation ARC pour la recherche sur le cancer (grant no PGA120150202355 to P.L.), the European Research Council (ERC Advanced grant no 268813 to P.L.).

\section{Author contributions}

Conceptualization and methodology: N.A., M.B., and P.L. Experimental investigation: N.A. and M.B. except mass spectrometry: L.K. and P.H. Formal analysis: N.A., Writing an original draft, supervision, and project administration: N.A. and P.L. Visualization:

N.A. Funding acquisition: P.L.

\section{Competing interests}

The authors declare no competing interests.

\section{Additional information}

Supplementary information The online version contains supplementary material available at https://doi.org/10.1038/s41467-021-25940-6.

Correspondence and requests for materials should be addressed to Nathalie Arquier.

Peer review information Nature Communications thanks the anonymous reviewers for their contributions to the peer review of this work. Peer review reports are available.

Reprints and permission information is available at http://www.nature.com/reprints

Publisher's note Springer Nature remains neutral with regard to jurisdictional claims in published maps and institutional affiliations.

(c) Open Access This article is licensed under a Creative Commons Attribution 4.0 International License, which permits use, sharing, adaptation, distribution and reproduction in any medium or format, as long as you give appropriate credit to the original author(s) and the source, provide a link to the Creative Commons license, and indicate if changes were made. The images or other third party material in this article are included in the article's Creative Commons license, unless indicated otherwise in a credit line to the material. If material is not included in the article's Creative Commons license and your intended use is not permitted by statutory regulation or exceeds the permitted use, you will need to obtain permission directly from the copyright holder. To view a copy of this license, visit http://creativecommons.org/ licenses/by/4.0/.

(C) The Author(s) 2021 